\section{Response of Fruit Bagging to Lignin Biosynthesis and Expression of Related Genes in Fruit Peel of Sand Pear (Pyrus pyrifolia Nakai) cv. Cuiguan}

\author{
Chun-hui Shi
}

College of Horticulture, Hebei Agricultural University, Baoding, Hebei, 071001, China; and Forest \& Fruit Tree Research Institute, Shanghai Academy of Agriculture Sciences, Shanghai, 201403, China

Xiao-qing Wang and Xue-ying Zhang

Forest \& Fruit Tree Research Institute, Shanghai Academy of Agriculture Sciences, Shanghai, 201403, China

\section{Lian-ying Shen \\ College of Horticulture, Hebei Agricultural University, Baoding, Hebei, 071001, China}

\section{Jun Luo}

Forest \& Fruit Tree Research Institute, Shanghai Academy of Agriculture Sciences, Shanghai, 201403, China

\section{Yu-xing Zhang \\ College of Horticulture, Hebei Agricultural University, Baoding, Hebei, 071001, China}

Additional index words. cork layer, full bloom, fruit bag, light quality, phenylalanine ammonia lyase, russeting

Abstract. This study explored the effects of different colored bags (blue, green, white, yellow, orange, and red) on russet deposition on the peel of semi-russet 'Cuiguan' pears 10 days after full bloom (DAFB). The process of russeting of the peel and structure of the cork layer were characterized by microscopy and scanning electron microscopy (SEM), followed by the detection of lignin and the activity of enzymes involved in lignin synthesis. The expression of cinnamate-4-hydroxylase, 4-coumarate:coenzyme A ligase, cinnamyl alcohol dehydrogenase, cinnamoyl-CoA reductase, and peroxidase, which were related to phenylalanine ammonia-lyase, was determined via real-time quantitative polymerase chain reaction. Russeting of the outer peel of 'Cuiguan' pear accumulated rapidly at 80 DAFB, and a positive relationship between the russet index and lignin content was observed. Red and infrared (IR) ray, partial far-IR light (600-800 $\mathrm{nm}$ ), and ultraviolet-A light $(350-400 \mathrm{~nm})$ promoted russeting in 'Cuiguan' pear peel, whereas green light decreased russeting, the russet index, enzymatic activities, and the expression levels of enzymes involved in lignin synthesis. Values of all these factors were higher for ' $C$ uiguan' pears in red bags than for those in bags of other colors. These findings suggested that spectral components affected the synthesis of lignin and the formation of fruit russet. Storage in green bags reduced russeting and improved fruit appearance.

\footnotetext{
Received for publication 2 July 2019. Accepted for publication 22 Aug. 2019

This study was supported by a grant from Shanghai Fruit Industry Technology System (SASI2018-7), Shanghai Agricultural Youth Talent Fund (SAY20161-20), This study was financed in part by the research and demonstration of efficient production technology of green pear orchard (SAS2019 1-3) and molecular mechanism of bud flowering in Eriobotrya japonica (Thunb.) Lindl (SAS2015 6-2-2). The authors thank Dr. Wei Jiang for the excellent technical support.

J.L. and Y.Z. are the corresponding authors. E-mail: zhangyuxing7199@163.com or zuowusuo (a)163.com.
}

Fruit color is a commercially important quality for sand pear (Pyrus pyrifolia Nakai). Sand pear can be divided into russet, semi-russet, and green types based on the color of the peel, which is typically composed of a cuticle layer, an epidermis cell layer, and a cork meristem (Wang et al., 2016). A green peel is formed by the accumulation of chlorophyll in epidermal cells, whereas a russet peel results from microscopic cracks and accumulation of suberin on the cell wall of the epidermal cell layers resulting from growth stresses and several endogenous factors (Graça and
Pereira, 2000; Khanal et al., 2012). Russet (semi-russeted) pears have an uneven, rough, and mottled brown appearance that will cause a decline in commercial quality. Therefore, reducing fruit russeting is a top priority among producers.

The suberification process is closely related to lignin and phenolic concentrations (Bernards, 2002; Graça and Santos, 2007), and it has been reported that lignin, cellulose, and hemicellulose contents are higher in russet peel than in green peel (Heng et al., 2014; Shi et al., 2019). Suberin is a macromolecular structure composed of the suberin poly-phenolic domain (SPPD) and the suberin polyaliphatic domain (SPAD), which are covalently linked by hydroxyferulic acid and its derivatives in a lignin-like manner (Meyer et al., 2011a, 2011b). Lignin, a primary component of plant cell walls, is an abundant natural polymer that is based on phenylpropane derivatives, and its biosynthesis has been shown to be closely associated with russeting in sand pear ( $\mathrm{Lu}$ et al., 2015).

Numerous enzymes are involved in the synthesis of lignin, including phenylalanine ammonia-lyase (PAL), 4-coumaric acid coenzyme A ligase (4CL), cinnamyl alcohol dehydrogenase (CAD), cinnamate-4-hydroxylase $(\mathrm{C} 4 \mathrm{H})$, cinnamoyl-CoA reductase (CCR), and peroxidase (POD) (Hu et al., 2017; Mertz-Henning et al., 2015; Shi et al., 2019). Most of the enzymes in the lignin metabolic pathway are induced by light at the transcriptional level, such as PAL, the activity of which is closely related to C4H, 4CL, CAD, and CCR (Ferrer et al., 2008; Hemm et al., 2004). In addition, PAL activity increases to varying degrees in Ginkgo biloba leaves and apple irradiated by red, white, blue, and strong ultraviolet lights (Liu and Cheng, 2003; Wang et al., 2000).

Numerous studies have shown how the synthesis of secondary metabolites related to the phenylpropanoid metabolic pathway in horticultural plants, such as flavonoids and phenols (Koyama et al., 2012; TomásBarberán and Espín, 2001), is influenced by light quality, but little research has focused on the regulation of lignin metabolism by light quality, and a clear understanding of how the phenylpropanoid metabolic pathway corresponds to lignin in pear fruit is lacking. Because fruit color is a highly desirable trait in pears, our objective was to examine the mechanism underlying russeting on the fruit peel of the 'Cuiguan' variety of sand pear, with the goal of forming the theoretical basis for directional control of fruit quality. It has been reported that different colored bags provided physical protection that could promote skin coloration of fruits by changing the natural light (Sharma et al., 2014a, 2014b). Specifically, we investigated how different light qualities induced by various light-filtering film bags of various colors affected key enzymes and genes involved in phenylpropanoid-ferulic acid lignin metabolism. 


\section{Materials and Methods}

Plant material and experimental treatments. Experiments were performed in an orchard located on the grounds of the Shanghai Jingguan Fruit Industry Co., Ltd. (lat. $30.92^{\circ} \mathrm{N}$, long. $121.47^{\circ} \mathrm{E}$; clay loam; annual average temperature, $15.7{ }^{\circ} \mathrm{C}$; annual rainfall, $1178.2 \mathrm{~mm}$ ) in Shanghai, China. Semi-russet Chinese sand pear ( $P$. pyrifolia 'Cuiguan') was used.

A total of five 12-year-old trees similar in size and fruit load and receiving sunlight uniformly were selected for the experiment. Approximately 120 fruitlets per tree were bagged using six colors of Kodak WRATTEN filter bags (blue, green, white, yellow, orange, and red) $10 \mathrm{~d}$ after full bloom (DAFB). A white bag was used as the control. The entire development period of 'Cuiguan' fruit is $120 \mathrm{~d}$. The trend of the russeting process was divided into two stages: sharply decreased period (10-70 DAFB) and steady changed period (80-120 DAFB). Sampling was performed every $10 \mathrm{~d}$ during the sharply decreased period, and sampling was performed every $20 \mathrm{~d}$ during the steady changed period. Three replicates were used for each treatment, and 30 pear fruits were randomly selected from 20 pear trees in each group. The outer peel of the fruit bellies was taken and immediately frozen in liquid nitrogen for extraction of RNA and enzyme activity determination. Spectrum parameters of filter bags were detected with a spectral radiometer (JETI Specbos 1211; JETI, Jena, Germany) under direct sunlight.

Evaluation of the russeting index. Russeting evaluations were based on the method described by Jones et al. (1991). Because of the high russeting coverage of the 'Cuiguan' pear peel, fruits were divided into eight grades according to the degree of rust: grade 0 (russet-free fruit), grade $1(1 \%$ to $5 \%$ russeting), grade $2(6 \%$ to $10 \%)$, grade 3 (11\% to $15 \%)$, grade $4(16 \%$ to $20 \%)$, grade 5 ( $21 \%$ to $40 \%)$, grade $6(41 \%$ to $60 \%$ ), grade 7 (61\% to $80 \%)$, and grade $8(81 \%$ to $100 \%)$. The russet index was calculated as follows:

$$
\begin{aligned}
& \text { Russet index }=\left[\sum(\text { grade } \times \text { number })\right] / \\
& (\text { total number of fruit } \times \text { highest grade }) \\
& \times 100 \%,
\end{aligned}
$$

and expressed as a percentage.

Cork layer structure. The tissues of the sand pear peels in the developmental phases (10-120 DAFB) were first examined with an electron microscope $(10 \times)$ (Leica M205 FA, Germany); then a scanning electron microscope was used to examine the tissue structure of russeting in more detail. Peels of 'Cuiguan' $(5 \mathrm{~mm} \times 5 \mathrm{~mm})$ pears at the mature stage were removed and fixed to the precooled sample base with frozen glue, after which the samples were inserted in liquid nitrogen mud at a temperature below $130 \mathrm{~K}$ $\left(-143.15^{\circ} \mathrm{C}\right)$ for rapid freezing and fixation (Quorum 2100 SEM freezing conveyor).
Next, under vacuum conditions, samples were transferred to a cold machine in a sample preparation chamber mounted on the SEM sample chamber port. Freezefracture, freeze-etching, and sputter coating were performed as needed. Finally, the shape and surface morphology of the russeted tissues on mature 'Cuiguan' pear peels were observed and photographed via SEM (S$3400 \mathrm{~N}$; Hitachi, Tokyo, Japan).

Lignin measurement of lignin content. Lignin content was determined based on the methods as previously described (Morrison, 1972) with some modification. Measurements of the lignin content were performed. First, $\approx 0.5 \mathrm{~g}$ of the peel material stored in liquid nitrogen at $-80{ }^{\circ} \mathrm{C}$ was removed and homogenized in $95 \%$ ethanol. Then, after centrifuging at $3000 g_{\mathrm{n}}$ for $7 \mathrm{~min}$, the precipitate was rinsed three times with $95 \%$ ethanol and an ethanol:n-hexane mixture at a ratio of $1: 2$, after which the precipitate was collected and dried. The dry matter was then dissolved in $25 \%$ bromoacetyl glacial acetic acid solution and incubated at $70{ }^{\circ} \mathrm{C}$ for 30 min. Approximately $0.9 \mathrm{~mL}$ of $\mathrm{NaOH}(2 \mathrm{M})$ was added to end the reaction. Finally, 0.1 $\mathrm{mL}$ of hydroxylamine hydrochloride $(7.5 \mathrm{M})$ was added to the reaction tube and diluted to $10 \mathrm{~mL}$ with glacial acetic acid. After centrifuging at $1000 g_{n}$ for $7 \mathrm{~min}$, absorbance of the resulting supernatant was measured at 280 $\mathrm{nm}$, and the absorption value at $280 \mathrm{~nm}$ per gram of fresh weight of 'Cuiguan' peel represented the lignin content.

Measurement of enzymatic activity. PAL activity was assessed following the procedures described by Villarreal et al. (2010). POD extraction was based on modified procedures described by Bestwick et al. (1998).

$4 \mathrm{CL}$ activity was evaluated using the methods described by Knobloch and Hahlbrock (1977). Additionally, CAD and C4H activity levels were assessed following the procedures described by Goffner et al. (1994) and Lamb and Rubery (1975), respectively. Enzymatic activity levels were expressed on a fresh weight basis (unit/g FW).

$R N A$ extraction and cDNA synthesis. Samples were obtained from the peel tissue of pears at 10-20 DAFB for all groups. Total RNA was extracted using a plant RNA kit (Omega Bio-Tek, Guangzhou, China) in accordance with the manufacturer's instructions. The first cDNA strand was reversetranscribed using a Revert Aid First Strand cDNA synthesis kit (Takara RR047A; Takara, Otsu, Shiga, Japan) in accordance with the manufacturer's instructions. For each time point of the groups, three batches of RNA were isolated as three biological repeats for separate cDNA synthesis.

Real-time quantitative polymerase chain reaction. The primer sets used in this study were designed by Primer 3 (http://primer3. sourceforge.net), with pear actin used as an internal control. Primer sequences of the target genes are shown in Supplemental Table 1. A quantitative polymerase chain reaction (qPCR) was performed with a realtime PCR instrument (Takara Bio USA,
Mountain View, CA). The reaction volume was $20 \mu \mathrm{L}$ and contained $2 \mu \mathrm{L}$ of cDNA, $2 \mu \mathrm{L}$ of primers, and $10 \mu \mathrm{L}$ of 29 SYBR Green PCR Master Mix (Roche Holding AG, Basel, Switzerland). The PCR protocols consisted of annealing at $94{ }^{\circ} \mathrm{C}$ for $5 \mathrm{~min}, 94{ }^{\circ} \mathrm{C}$ for 15 $\mathrm{s}$, and 40 cycles at $60^{\circ} \mathrm{C}$ for $1 \mathrm{~min}$ each. Each run contained a control without template for each primer pair. The results were analyzed using the 2-DCT method (Willems et al., 2008), and all relative intensities were calculated from standard curves.

Statistical analysis. One-way analysis of variance was conducted to detect significant differences; least significant differences $(\alpha=$ 0.05 ) were calculated for mean separations using SPSS; furthermore, all statistical analyses were performed using SPSS software (version 13.0; SPSS Inc., Cary, NC).

\section{Results}

Effects of different colored bags on light quality. To evaluate the effects of light on the coloration of sand pear peel, pears were treated with filtered fruit bags of different colors. Filter bags significantly affected the spectral parameters and transmittance of sunlight. As shown in Table 1 and Supplemental Fig. 1, all six colors significantly changed the solar spectrum within a range of 350-800 $\mathrm{nm}$. White light contains many monochromatic light sources and is the representative irradiance spectrum most similar to sunlight. Ultraviolet transmittance $(2.03 \%)$ was higher in the white bags than in the five monochromatic light filter treatment bags (yellow, orange, red, green, and blue). Compared with the white treatment (control), the transmission ratio of ultraviolet-A light (wavelengths of 350 $400 \mathrm{~nm}$ ) was decreased to varying degrees in all of the light filter bags. Of the five monochromatic light filter bags, ultravioletA transmittance was the lowest in green filter bags $(0.92 \%)$ and highest in blue filter bags $(1.96 \%)$; in the spectral wavelength range of 400-600 nm, transmittance was the highest in green and lowest in red filter bags; and in the spectral wavelength range of 600 $800 \mathrm{~nm}(\mathrm{Pr}+\mathrm{Pfr})$, transmittance was the highest in red $(79.65 \%)$ and lowest in white $(58.33 \%)$ filter bags. The ratio of red light to far-IR ray transmittance (R/FR) of the controls was close to 1.0 , as were the $\mathrm{R} / \mathrm{FR}$ of the yellow, orange, and red filter bags; in contrast, the R/FR of the green and blue filter bags were $<1$, at 0.78 and 0.56 , respectively, and decreased light transmittance to within the range of $45.9 \%$ to $70 \%$.

Fruit peel appearance and russeting microstructure. As shown in Fig. 1, the occurrence time and area of russeting differed greatly among the color groups. In the white (control) group (ultraviolet-A transmittance $=2.03 \% ; \operatorname{Pr} / \mathrm{Pfr}=\approx 1)$, russeting formed at $10 \mathrm{DAFB}, \approx 10 \mathrm{~d}$ earlier than in the other five color groups; in the yellow, orange, red, and control groups $(\mathrm{Pr} / \mathrm{Pfr}=\approx 1)$, russeting changed significantly during the fruit development, russeting (20 DAFB), flaky 
Table 1. Ratio of representative irradiance spectrum in different color treatments.

\begin{tabular}{|c|c|c|c|c|c|c|c|c|}
\hline $\begin{array}{l}\text { Transmission } \\
\text { spectrum }\end{array}$ & Color of bag & $\begin{array}{c}\text { UV-A } \\
(350-400 \mathrm{~nm}) / \%\end{array}$ & $\begin{array}{c}\text { Blue } \\
(400-500 \mathrm{~nm}) / \%\end{array}$ & $\begin{array}{c}\text { Green } \\
(500-600 \mathrm{~nm}) / \%\end{array}$ & $\begin{array}{c}\text { Red } \\
(600-700 \mathrm{~nm}) / \%\end{array}$ & $\begin{array}{c}\text { Far-red } \\
(700-800 \mathrm{~nm}) / \%\end{array}$ & $\mathrm{Pr} / \mathrm{Pf}$ & $\mathrm{Pr}+\mathrm{Pfr} / \%$ \\
\hline & $\begin{array}{l}\text { White } \\
\text { (Control) }\end{array}$ & 2.03 & 15.45 & 24.19 & 28.98 & 29.35 & 0.99 & 58.33 \\
\hline & Yellow & 1.46 & 8.63 & 24.79 & 32.25 & 32.89 & 0.98 & 65.13 \\
\hline & Red & 1.31 & 5.76 & 13.28 & 38.31 & 41.35 & 0.93 & 79.65 \\
\hline & Green & 0.92 & 13.42 & 25.10 & 26.41 & 34.15 & 0.77 & 60.56 \\
\hline
\end{tabular}

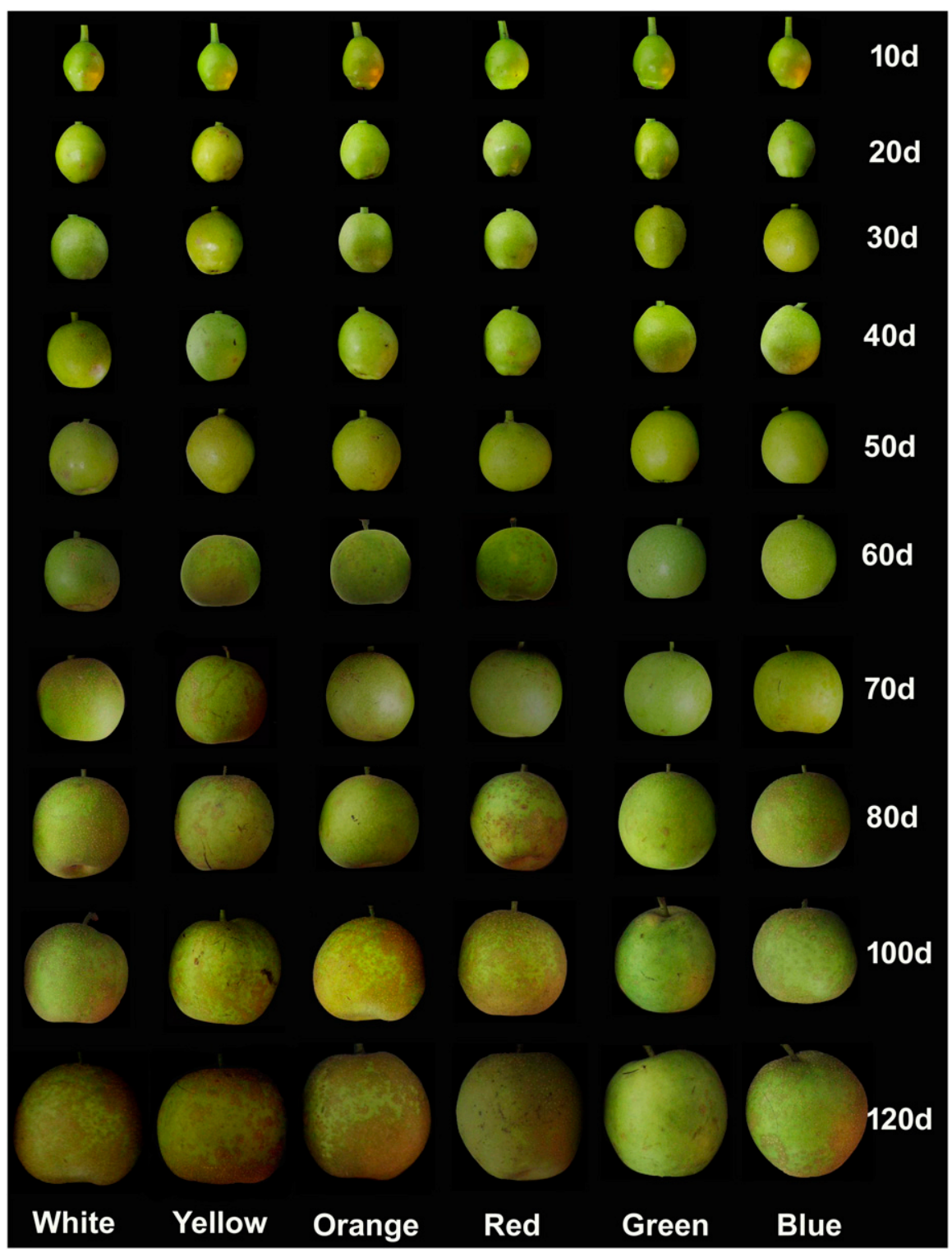

Fig. 1. Phenotypic traits of 'Cuiguan' pear covered with bags of different colors at different stages of days after full bloom (DAFB). 'Cuiguan' pears were divided into the six groups: white (control), yellow, orange, red, green, and blue. russeting (40 DAFB), and rapid expansion (60 DAFB) periods. With the blue (ultravioletA transmittance $=1.96 \% ; \mathrm{Pr} / \mathrm{Pfr}=0.57$ ) and green (ultraviolet-A transmittance $=0.92 \% ; \mathrm{Pr}$ / Pfr $=0.78)$ filter bags, russeting appeared later and covered a smaller area than what occurred with the other colors of bags; furthermore, with green bags, the russeting formation period was $40 \mathrm{~d}$ later than that of the control group.

Fig. 2A shows the change in the russet index, which shows how the extent of coverage of russet increased as lignin content gradually increased with fruit development (Fig. 2B). The russet index was counted in all the samples from $10 \mathrm{DAFB}$, except for those from the green and blue filter groups. In all groups, the russet index increased quickly and peaked at $80 \mathrm{DAFB}$; after which, it remained stable throughout the mature stage. The index of the red filter group $(88.33 \%)$ was highest at the mature stage, followed by the white (control) group $(87.50 \%)$; these were significantly different from those of the other four groups $(P<0.05)$, and the outer skins of fruits in these groups were covered with sheets of dark brown cork layer. The order was as follows (high to low): natural red $>$ white (control) $>$ yellow $>$ orange $>$ blue $>$ green. There was a significant difference between the two treatments in the other groups $(P<0.05)$. The green group had the lowest russet index, which was $45.8 \%$ lower than that of the control.

The peels of six color groups for 'Cuiguan' were observed with respect to microstructure and submicrostructure. We found that the phellem cells were initially suberizated around the lenticels. Microstructures of peels collected from fresh 'Cuiguan' pears from 10 120 DAFB are shown in Fig. 3. Overall, peel russeting was significantly lower in the green group than in the other groups, and the fruits showed tiny russet spots at the maturation stage. Specifically, in the white (control) and red groups, suberization formed around the lenticels at 10 DAFB and slowly expanded, 

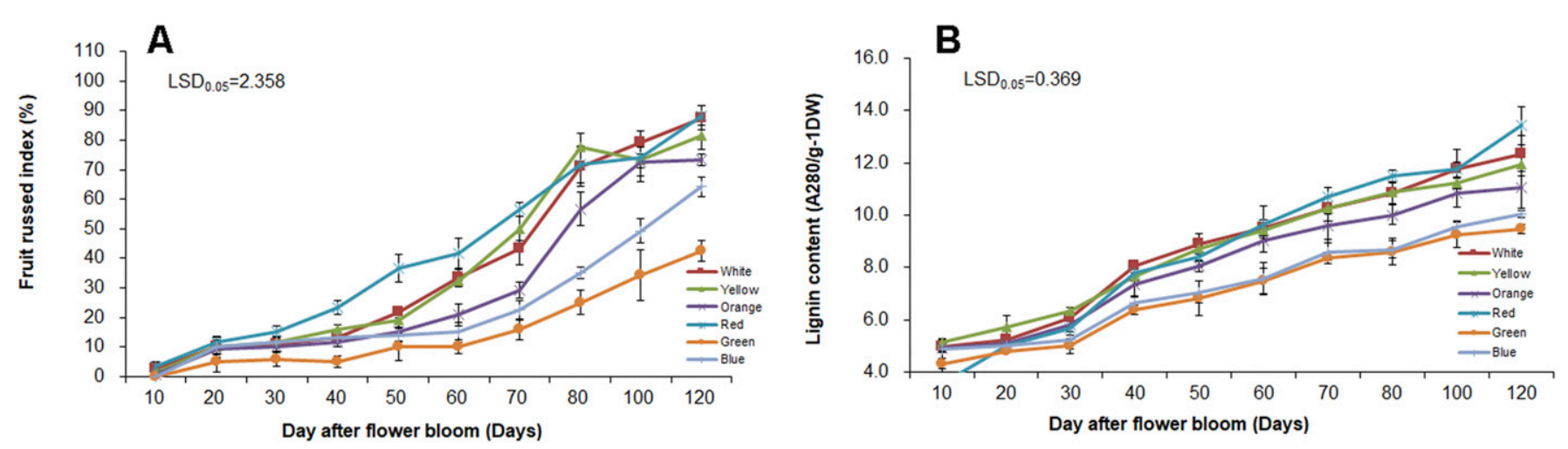

Fig. 2. Effects of different colored bags according to the russet index (A) and lignin content (B) in 'Cuiguan' pear at different stages of days after full bloom (DAFB). Data are expressed as means \pm SD of three biological replicates.

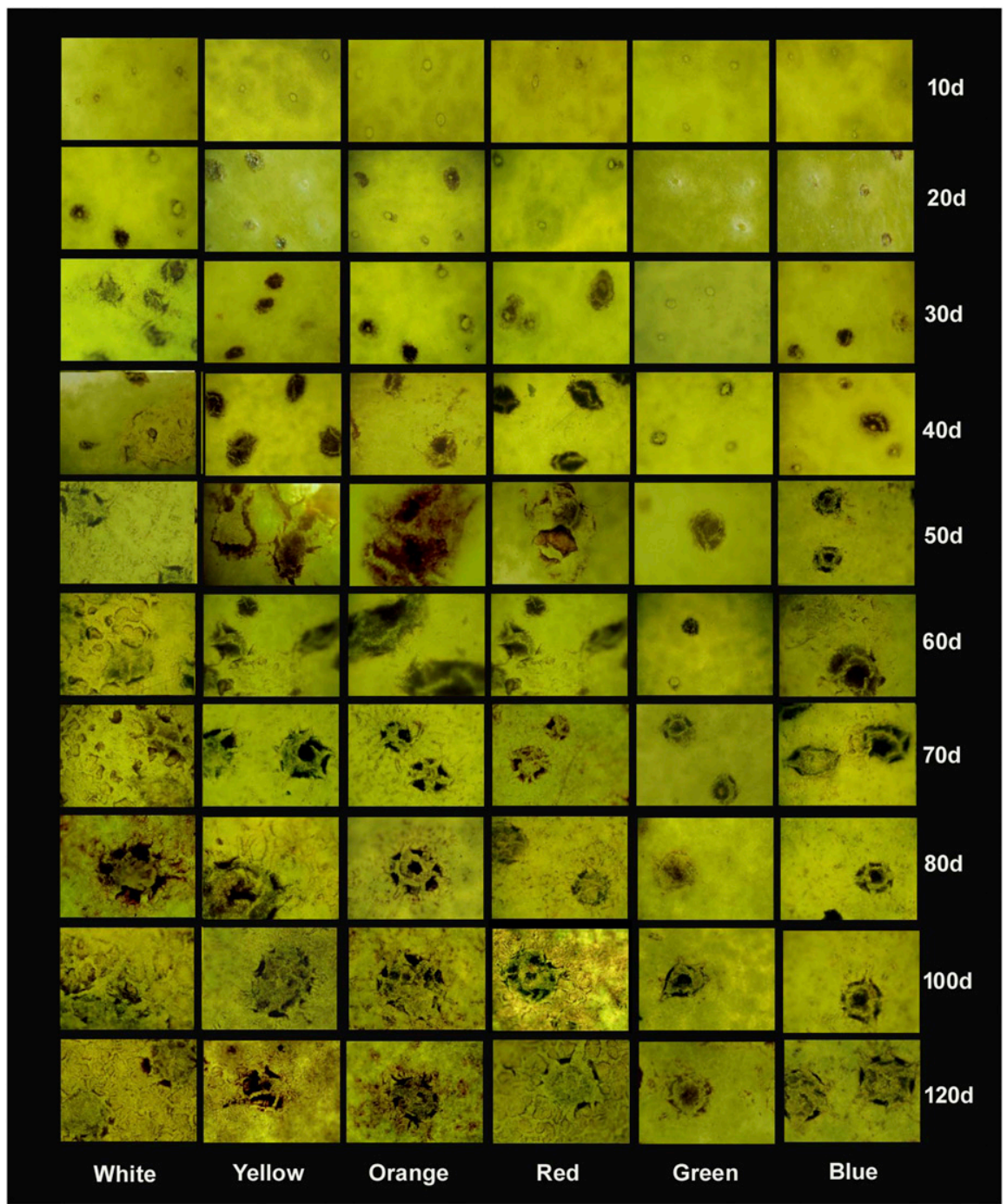

Fig. 3. Microstructure comparison of 'Cuiguan' peel with different colored bags at different stages of days after full bloom (DAFB) (magnification 20x).

particularly at 30 DAFB. In contrast, in the green and blue groups, suberization appeared at 40 and 20 DAFB, respectively. At 40 DAFB, the outermost layer of the stratum corneum in the peel of fresh 'Cuiguan' pears was cracked and fragmented, with epidermal cells showing significant phellem layer forma- tion in the white (control), yellow, orange, and red groups. Moreover, the degree of suberization was more intense during the late fruit ripening stage in these groups. Submicrostructures of the outer epidermis and longitudinal section of the peel are shown in Fig. 4. It can be seen that the degree of cutin rupture and suberization was higher in the white (control) group and red group than in the other groups, whereas the keratinocyte membrane was complete and there was a smaller cork layer on the peel surface of 'Cuiguan' pears stored in green filter bags.

Analysis of lignin content and enzymatic activity in 'Cuiguan' peel. We found that the overall trends of the lignin content and fruit russet index were similar during all developmental stages. The results showed that the correlation coefficient for the peel russet index and lignin content was 0.928 , which was very significant. To characterize the light quality regulation of lignin biosynthesis, we measured both the lignin increments and the activity levels of the enzymes PAL, CAD, $\mathrm{C} 4 \mathrm{H}, 4 \mathrm{CL}$, and POD in the peel during the period from 10-120 DAFB. As can be seen in Fig. 2B, the lignin content continually increased over the development of the pear fruit; this increase was higher in fruits harvested at 40 DAFB and tended to be stable at the mature stage. Lignin content occurred in the following order (high to low): red $>$ white (control) $>$ yellow $>$ orange $>$ blue $>$ green . The red filter group had the highest lignin content, which was significantly higher than that of the white (control) group. The green filter group had the lowest content, which was significantly decreased by $23.32 \%$ compared with that of the control treatment group.

The synthesis of lignin monomers is performed via the phenylpropionic acid pathway. To learn more about light quality regulation of lignin synthesis, we further explored the link between the dynamic variables of lignin and the changes in key enzymes in lignin biosynthesis. Fig. 5A shows the incremental increase of lignin content in the peel of 'Cuiguan' pear, with content increasing rapidly from 30-40 DAFB, which might be associated with the rapid division of fruit cells that occurs after this phase. The cumulative incremental increase in lignin content was highest in the red group, but it was not significantly higher than that in the white (control) group; however, it was significantly higher than that in the other four color groups. The lignin increment of the green group was significantly lower than that of the other groups during the late stage of fruit development $(<60.87 \%$ and $86.65 \%$, respectively) 

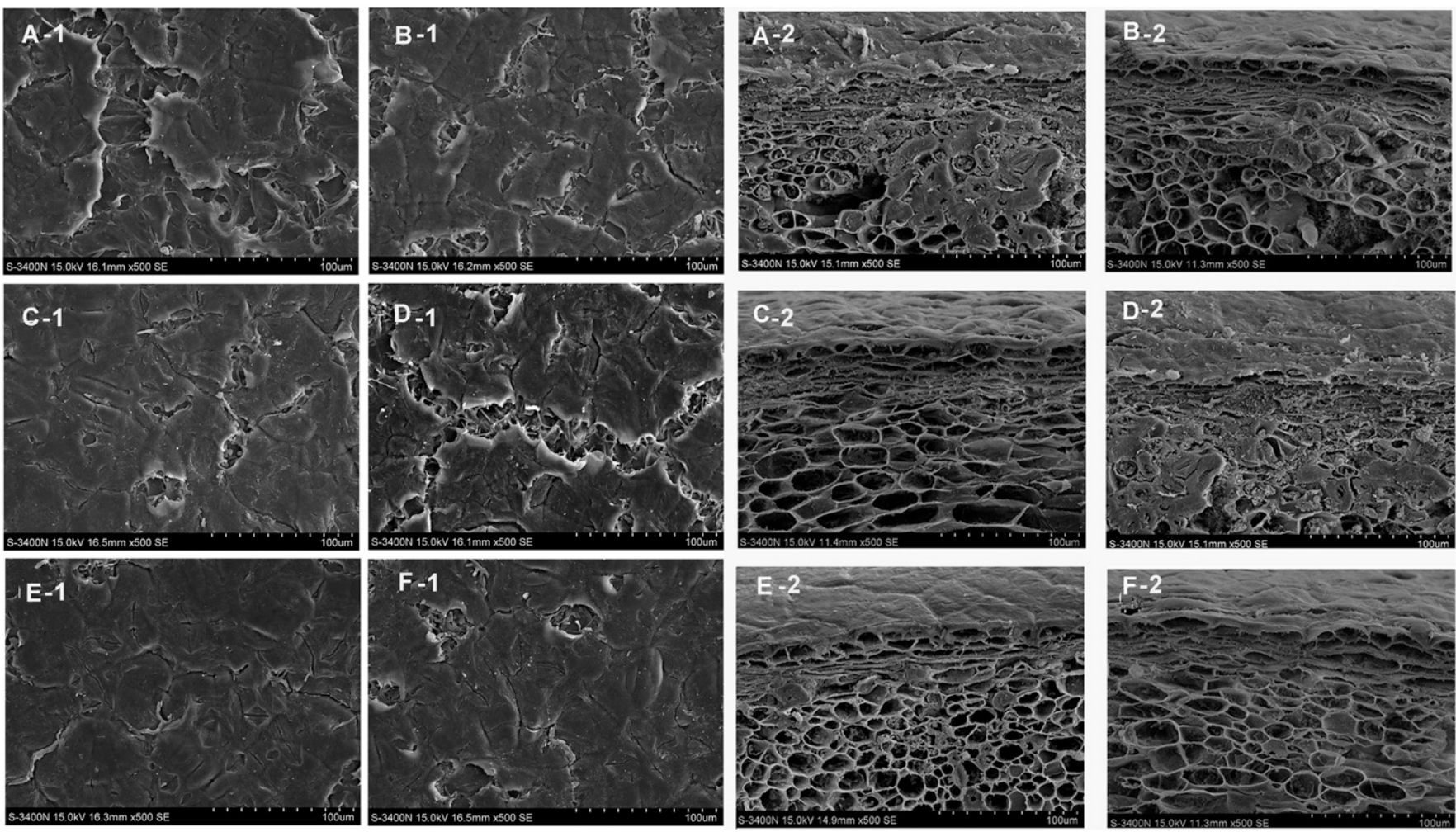

Fig. 4. The ultra-microstructures on the surface (left panel) and longitudinal sections (right panel) of the peel of 'Cuiguan' with different colored bags at $80 \mathrm{DAFB}$ (scanning electron microscopy freezing conveyor 500×). (A-1) Control (white) (on the peel surface). (B-1) Yellow bags (on the peel surface). (C-1) Orange bags (on the peel surface). (D-1) Red bags (on the peel surface). (E-1) Green bags (on the peel surface). (F-1) Blue bags (on the peel surface). (A-2) Control (white) (from peel longitudinal section). (B-2) Yellow bags (from peel longitudinal section). (C-2) Orange bags (from peel longitudinal section). (D-2) Red bags (from peel longitudinal section). (E-2) Green bags (from peel longitudinal section). (F-2) Blue bags (from peel longitudinal section).

compared with the control and red groups. Fig. 5B-D illustrates the activities of the enzymes PAL, C4H, and 4CL. The change trends of these three enzyme activities were consistent with those of the highest enzyme activities from 30-40 DAFB. Fig. 5E and $F$ show that the activity of POD was also consistent with the three aforementioned enzymes during the early stage of fruit development. The activities of CAD and POD enzymes were involved in the final phases of lignin synthesis, which peaked at 70-80 DAFB. Based on the correlation analysis shown in Fig. 6, there was a highly significant correlation between the increase in lignin content and the activities of five key enzymes (PAL, C4H, 4CL, CAD, and POD) in the phenylalanine pathway. The increment of lignin was highly correlated with PAL and POD, with correlation coefficients of 0.520 and 0.608 .

Gene expression of functional enzymes in lignin synthesis. Regulation of the expression of the genes coding for the key enzymes involved in lignin biosynthesis was also examined. As can be seen in Fig. 7, the genes PpyPAL, PpyC4H, Ppy $4 C L$, and PpyPOD were all highly expressed at 40 DAFB; however, expression levels began to decline thereafter. Expressions of these five genes in the red group were significantly higher than those in the other groups, and their expressions in the green group were significantly lower than those in the other groups. PpyCAD and $P p y C C R$ gradually decreased during fruit de- velopment. However, the difference between them was that the expression of PpyCAD showed an increase during the late stage of fruit development (60 DAFB) and then declined. In contrast, PpyPAL expression was significantly higher in the red group than in the other treatments at $40 \mathrm{DAFB}$, and it was 3.43 times and 85.18 times higher than in the white (Control) and green groups, respectively. The $P p y C 4 H$ expression level initially increased and then decreased (20-120 DAFB), with the highest value in the red group, with the following order (high to low): red $>$ orange $>$ white $($ control) $>$ blue $>$ yellow $>$ green. The expression of Ppy $4 C L$ initially increased and then decreased at the whole fruit development stage; its expression was significantly higher in the red group and blue group than in the white (control), and both were 1.10 times at 40 DAFB. Unlike the other enzymes, expression of $P p y C C R$ was higher in the white and green groups than in the other groups (Fig. 7F). As the fruit development russet index increased, the expression level of PpyCCR showed a declining trend and the green group with the lowest russet index had the highest expression level compared with the other groups.

\section{Discussion}

The peel of semi-russet pear was attached to the irregular lignified cork coat, which was a bad quality trait of this variety because this coat would be affected by the environment or the cultivation techniques. Bagging is often used to protect fruits such as apple and Asian pear (Huang et al., 2009; Ju, 1998; Santos and Wamser, 2006). The fruit bag is supposed to prevent rainwater and change the light (Wang et al., 2014). Shi et al. (2019) revealed that the rain shelter might affect phenylalanine lignin monomer synthesis and decreased peel russet accumulation in semi-russet pear. Previous research has largely focused on uncovering the physiological, biochemical, and molecular mechanisms responsible for russeting; however, the way in which light quality influences this process in sand pear is largely unknown. Here, we compared fruit kept in fruit bags of various colors to explore how differences in light quality affect russeting in the 'Cuiguan' pear. Russeting of the sand pear peel is presumed to be a typical feature of the fruit in response to adverse external environmental conditions (Wang et al., 2016). Russeting is associated with suberin deposition (Khanal et al., 2012), and the SPPD component of suberin is the result of polymerization of hydroxybutanoic acid and lignin analogues, indicating that russeting is strongly associated with lignin accumulation (Heng et al., 2014; Kalisz et al., 2015). Wang et al. (2014) demonstrated the differences in suberin, cutin, wax, and lignin biosynthesis between russet and green peels of sand pear. In addition, it has been shown that bagging during the growth period significantly decreases the russet incidence in the 

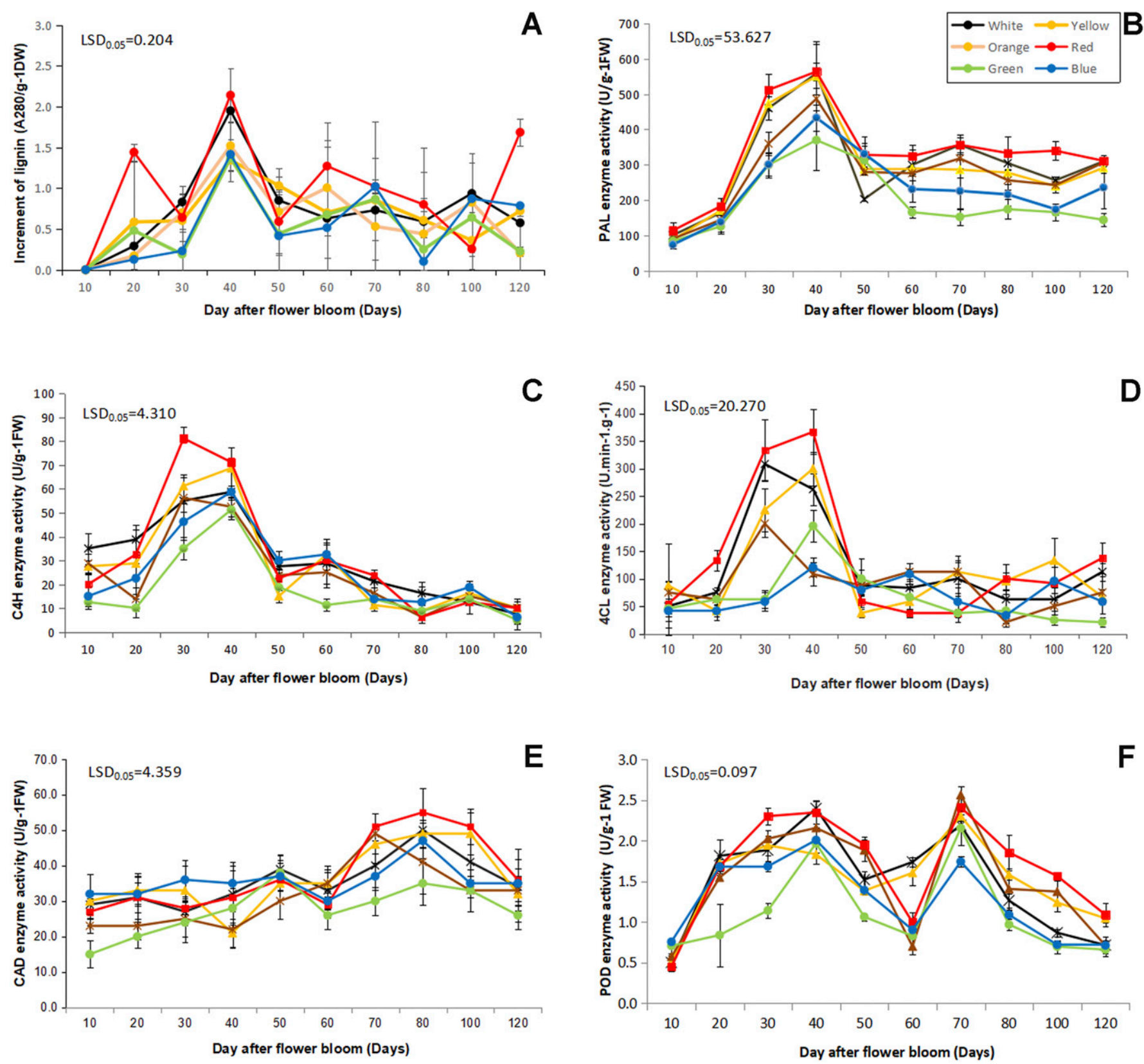

Fig. 5. Activity levels of enzymes involved in lignin synthesis in 'Cuiguan' pears covered with different colored filter film bags. (A) Increment of lignin. (B) Phenylalanine ammonia-lyase (PAL). (C) Cinnamate-4-hydroxylase $(\mathrm{C} 4 \mathrm{H})$. (D) 4-coumarate-coenzyme A ligase (4CL). (E) Cinnamyl alcohol dehydrogenase (CAD). (F) Peroxidase (POD). The x-axis represents DAFB and the y-axis represents enzyme activity level.

peel, as well as latent infection and decay of fruits, whereas degreening of fruit peels is accelerated (Jun et al., 2013). The results of our study provided further evidence that the accumulation of russeting in the peel of 'Cuiguan' pears was significantly correlated with lignin content, and that the colored fruit bags had different effects on russeting in this pear variety that were consistent with those reported by previous studies.

From $60-80$ DAFB, as the 'Cuiguan' pear fruits expanded, the russeting on the peel accumulated rapidly, indicating that 80 DAFB was the key period of russet progression. It has also been reported that russeting spread rapidly after 80 DAFB, which may be because of the involvement of cork cambium in the synthesis of the cork layer (Wang et al., 2014). In addition, microscopy and SEM were used to detect differences in tissue structure between the russet and green peels of sand pear. Compared with the white (control) group, the red group cork layer accumulated quickly, and there was a difference in the accumulation of cork following bagging (i.e., different light quality treatments). Fruit bags of different color not only changed the spectral composition of incoming light but also caused differential reductions in light transmittance. In this study, we did not find a direct link between light transmittance and fruit russeting. Light is one of the most important environmental factors affecting plant growth and secondary metabolism, and light quality has a greater influence on plant secondary metabolism compared with light intensity (Keller and Hrazdina, 1998).
Lignin, flavonoids, pigments, and phenolic compounds are all products of the phenylpropane metabolic pathway, and light has a key role in their metabolism (Dixon and Paiva, 1995; Lefsrud et al., 2008). Generally, concentrations of plant secondary metabolites increase with increasing light quality within a certain range (Beggs and Wellmann, 1994; Christie and Jenkins, 1996). Zhang et al. (2012) reported that ultraviolet$B$ affected the metabolism of anthocyanin in red sand pear after harvest. In our study, the spectral composition of the white treatment was close to that of natural light, the red light filter group was 1.3 times the irradiance of the white light group in the red light band of $600-700 \mathrm{~nm}$, and the russet index of the 'Cuiguan' in the red filter treatment was 


\section{Correlation}

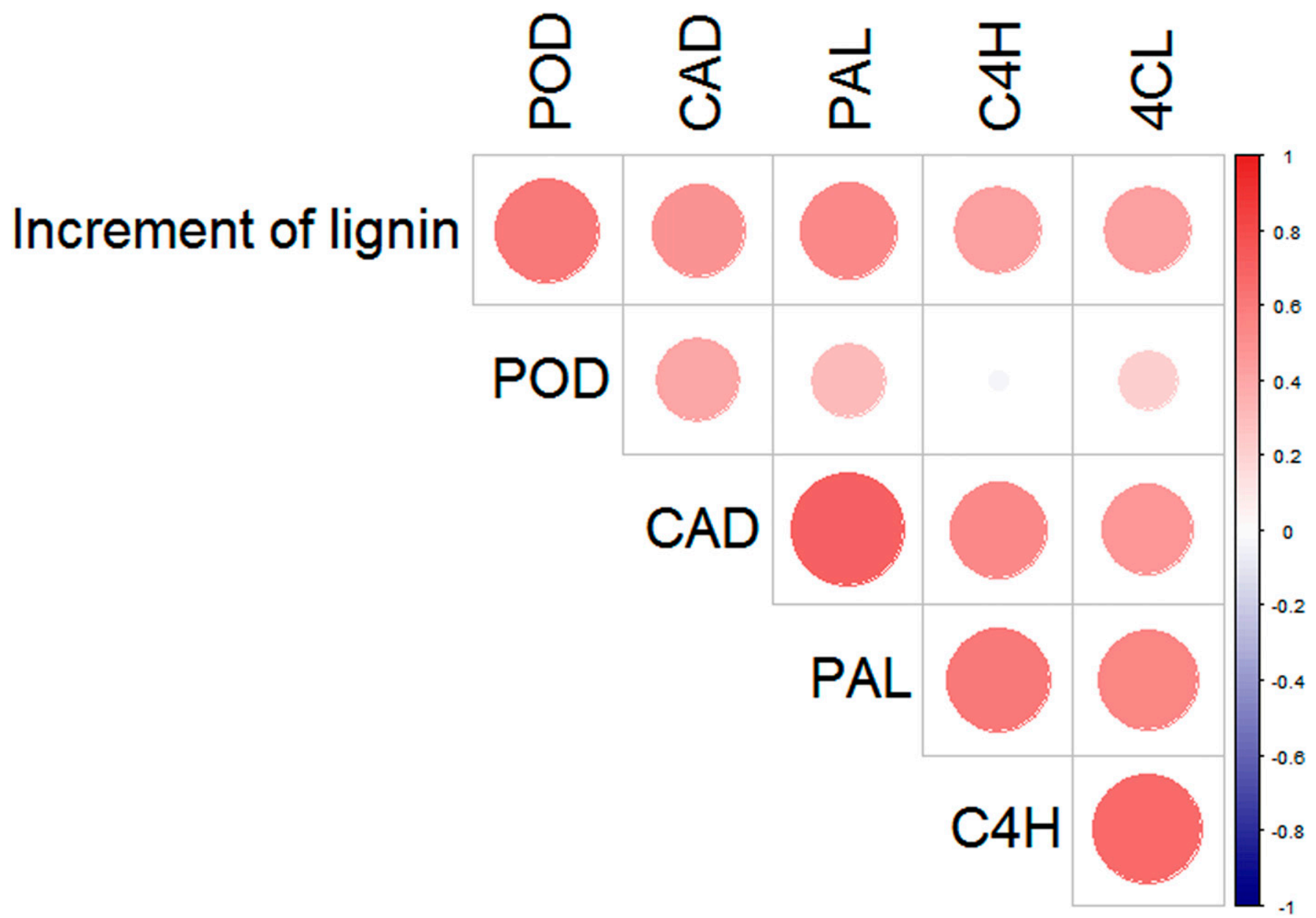

Fig. 6. Correlation analysis between lignin increments and key enzyme activities of lignin synthesis.
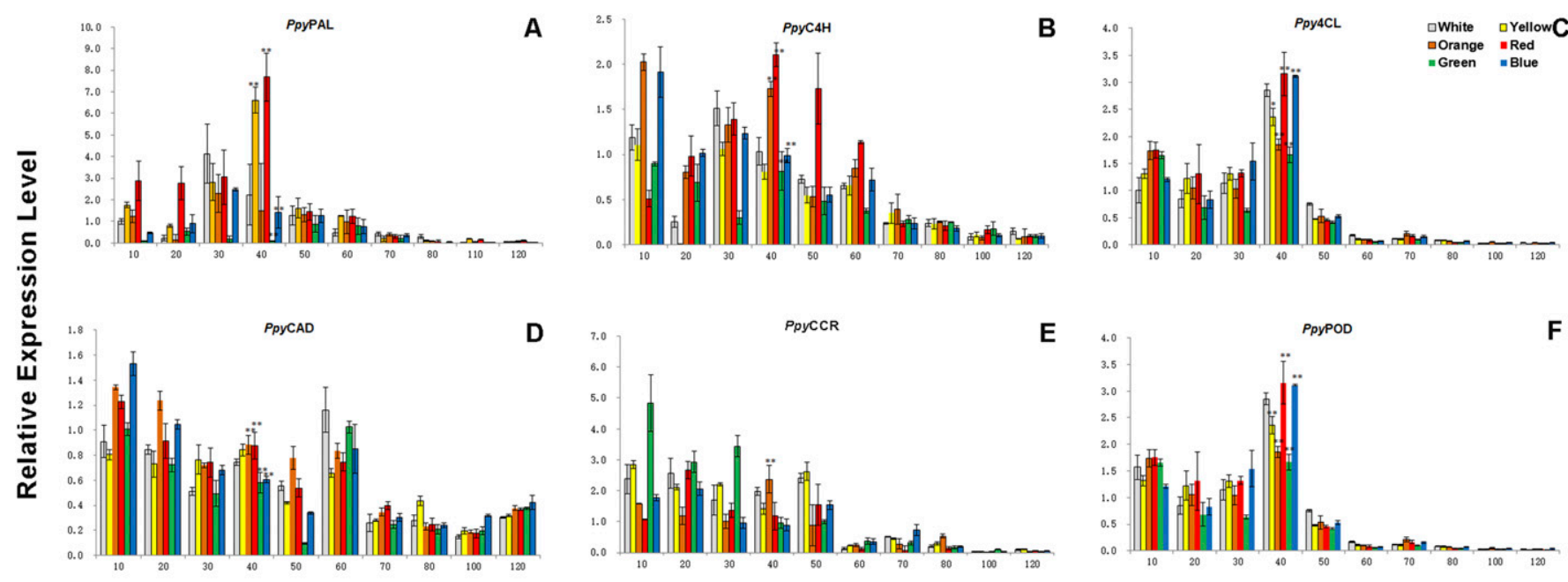

Day after flower bloom (Days)

Fig. 7. Expression levels of enzymes involved in lignin synthesis in 'Cuiguan' pear. (A) Phenylalanine ammonia-lyase $(P p y P A L)$. (B) Cinnamate-4-hydroxylase $(P p y C 4 H)$. (C) 4-coumarate-coenzyme A ligase $(P p y 4 C L)$. (D) Cinnamyl alcohol dehydrogenase (PpyCAD). (E) Cinnamoyl-CoA reductase $(P p y C C R)$. (F) Peroxidase $(P p y P O D)$. The $\mathrm{x}$-axis represents DAFB and the y-axis represents relative expression levels.

higher than that of the white (control). Although we speculated that the synthesis of lignin in the peel would be reduced as light intensity weakened, we did not find a direct link between light transmittance and fruit russeting. The effect of light quality on the production of phenylpropanoid metabolites was more complicated. At present, three types of photoreceptors are known, one of which is the phytochrome receptor type of red light and far red light, and the second of which is the ultraviolet-A/blue light 
receptor type that senses blue light and ultraviolet-A cryptochromes and phototropins (Heijde and Ulm, 2012). Because it is the most important signaling molecule, light has a major role in plant growth and development, especially in lignin synthesis. Whiting and Goring (1982) found that the lignin content of wheat cell walls increased following intermittent treatment with red light and far red light (Whiting and Goring, 1982). As a signal molecule, light controls hexoses and circadian clocks, and it has been shown to affect lignin synthesis in Arabidopsis at the transcriptional level (Rogers et al., 2005). Zhang et al. (2017) reported that exposure to light activated the phenylpropanoid metabolic pathway in plants and, consequently, lignin, tea catechin, and anthocyanin anabolism were strengthened. The results of these and other studies suggested that regulation of the plant secondary metabolite content by altering light quality conditions was feasible. This was supported by the results of our study, which showed that after changing solar spectrum components using filter bags of different colors, the green groups had significantly lower lignin content compared with the other groups. Red and far-IR light in the ranges of $600-800 \mathrm{~nm}$ and 350-400 nm ultraviolet-A light, respectively, promoted russet spread in the peel of 'Cuiguan' pears.

PAL, an inducing enzyme affected by light, acts as a general switch for phenylpropanoid metabolism and is the first ratelimiting enzyme of this process. Red, white, blue, and strong ultraviolet lights induce PAL at the transcriptional level, causing different degrees of PAL activity (Lafuente et al., 2003; Leyva et al., 1995; Sanchezballesta et al., 2000; Teklemariam and Blake, 2004). Franke et al. (2000) inhibited PAL or C4H in transgenic tobacco and found that concentrations of Klason's lignin decreased, suggesting that the lignin monomer synthesis might have a specific branch of $G$ or $S$ at the entrance of the phenylpropanoid pathway (Franke et al., 2000). In the present study, lignin content in the peel of pears undergoing green bag treatment was significantly lower than that of those undergoing the other treatments, and the levels of PpyPAL (transcription level) and PAL (enzyme activity) were lower as well. Under the action of the PAL enzyme, L-phenylalanine forms transcinnamic acid, which is catalyzed by a series of enzymes such as cinnamic acid-4hydroxylase to form its hydroxy derivative or methoxy derivative. These derivatives are then catalyzed by 4CL to form the corresponding coenzyme A, thereby entering the synthesis of the phenylpropane derivative (Blount et al., 2000; Jia et al., 2008; Xu et al., 2011). CAD catalyzes the synthesis of lignin monomers; based on $\mathrm{CAD}$, various cinnamaldehydes (coumarin, mustard aldehyde, coniferaldehyde, and others) are reduced to cinnamyl alcohols (coumarin, coniferyl alcohol, mustard alcohol, and others), which are precursors of lignin monomers (Fornalé et al., 2012; Sibout et al., 2005). In the present study, both the russet index and lignin content increased with higher proportions of far-IR light $(\mathrm{Pr} / \mathrm{Pfr}=$ $\approx 1$ ), and activity levels of PAL, 4CL, C4H, $\mathrm{CAD}, \mathrm{CCR}$, and POD enzymes were higher with the red and white light treatments than with the green treatment. Combined with the results of the qPCR, this suggested that the induced activity of enzymes involved in the synthase of lignin occurred at the transcriptional level, which was consistent with the results of Zhang et al. (2012). It is likely that the red filter bag increased the ratio of red light to IR ray, inducing a mediated reaction of the phytochrome $\mathrm{Pr} / \mathrm{Pfr}$ photoreceptor, which regulated the activity of the phenylpropanoid metabolism enzyme by regulating PAL, leading to the derivatives in the lignin synthesis process being more widely distributed in the pear peel. Our results showed that mRNA expression at the transcriptional level of PpyPAL, PpyC4H, Ppy $4 C L$, and PpyPOD had good consistency with their corresponding enzyme activity. Interestingly, the expression of PpyCAD was opposite that of CAD enzyme activity and the increment of lignin content. Although previous studies of CAD and CCR differed, some researchers have reported that with the reduction of CAD and CCR activity, expression of lignin precursors decreases and cell wall loss leads to russeting; however, other researchers have reported that these genes are inhibited with expression but found higher lignin content in russet pear and apple (Bernards, 2002; Bernards et al., 2000; Legay et al., 2015; Moura et al., 2010; Wang et al., 2014). Therefore, in addition to participating in lignin synthesis, CAD and CCR might also have their own independent ways of responding to the light environment. In addition, ultraviolet-A light promoted the synthesis of lignin in the peel of sand pear; however, the specific underlying mechanism was not identified and required further study.

In this study, the russet response in 'Cuiguan' pear was affected by light quality. Russet spread was least in pears kept in green filter bags, most likely because of the significantly lower activity of enzymes involved in lignin biosynthesis and the regulatory genes PpyPAL, PpyC4H, Ppy $4 C L, P p y C A D$, and $P$ pyPOD in these fruits, which reduced lignin content and, consequently, the russet index. Our study informed future research aimed at gaining a better understanding of the biosynthesis and regulation of russet deposits, which might lead to the development of new technologies for improving the color and quality of sand pear fruits.

\section{Literature Cited}

Bernards, M.A., L.M. Susag, D.L. Bedgar, A.M. Anterola, and N.G. Lewis. 2000. Induced phenylpropanoid metabolism during suberization and lignification: A comparative analysis. J. Plant Physiol. 157:601-607.

Bernards, M.A. 2002. Demystifying suberin. Can. J. Bot. 80:227-240.

Beggs, C.J. and E. Wellmann. 1994. Photocontrol of flavonoid biosynthesis, p. 733-751. In: R.E. Kendrick and G.H.M. Kronenberg (eds.). Pho- tomorphogenesis in plants. Springer, Dordrecht.

Bestwick, C.S., I.R. Brown, and J.W. Mansfield. 1998. Localized changes in peroxidase activity accompany hydrogen peroxide generation during the development of a nonhost hypersensitive reaction in lettuce. Plant Physiol. 118:1067-1078

Blount, J.W., K.L. Korth, S.A. Masoud, S. Rasmussen, C. Lamb, and R.A. Dixon. 2000. Altering expression of cinnamic acid 4-hydroxylase in transgenic plants provides evidence for a feedback loop at the entry point into the phenylpropanoid pathway. Plant Physiol. 122:107-116.

Christie, J.M. and G.I. Jenkins. 1996. Distinct UV$\mathrm{B}$ and UV-A/blue light signal transduction pathways induce chalcone synthase gene expression in arabidopsis cells. Plant Cell 8:1555-1567.

Dixon, R.A. and N.L. Paiva. 1995. Stress-induced phenylpropanoid metabolism. Plant Cell 7:10851097.

Ferrer, J.L., M.B. Austin, C. Stewart, and J.P. Noel. 2008. Structure and function of enzymes involved in the biosynthesis of phenylpropanoids. Plant Physiol. Biochem. 46:356-370.

Fornalé, S., M. Capellades, A. Encina, K. Wang, S. Irar, C. Lapierre, K. Ruel, J.P. Joseleau, J. Berenguer, P. Puigdomènech, J. Rigau, and D. Caparrós-Ruiz. 2012. Altered lignin biosynthesis improves cellulosic bioethanol production in transgenic maize plants down-regulated for cinnamyl alcohol dehydrogenase. Mol. Plant 5:817-830.

Franke, R., C.M. Mcmichael, K. Meyer, A.M Shirley, J.C. Cusumano, and C. Chapple. 2000. Modified lignin in tobacco and poplar plants over-expressing the Arabidopsis gene encoding ferulate 5-hydroxylase. Plant J. 22:223234.

Goffner, D., M.M. Campbell, C. Campargue, M. Clastre, G. Borderies, A. Boudet, and A.M. Boudet. 1994. Purification and characterization of cinnamoyl-coenzyme A:NADP oxidoreductase in Eucalyptus gunnii. Plant Physiol. 106:625-632.

Graç, J. and H. Pereira. 2000. Methanolysis of bark suberins: Analysis of glycerol and acid monomers. Phytochem. Anal. 11:45-51.

Graça, J. and S. Santos. 2007. Suberin: A biopolyester of plants' skin. Macromol. Biosci. 7:128-135.

Heijde, M. and R. Ulm. 2012. UV-B photoreceptormediated signalling in plants. Trends Plant Sci. 17:230-237.

Hemm, M.R., S.D. Rider, J. Ogas, D.J. Murry, and C. Chapple. 2004. Light induces phenylpropanoid metabolism in Arabidopsis roots. Plant J. 38:765-778

Heng, W., L. Liu, M.D. Wang, B. Jia, P. Liu, Z.F. Ye, and L.W. Zhu. 2014. Differentially expressed genes related to the formation of russet fruit skin in a mutant of 'Dangshansuli' pear (Pyrus bretchnederi Rehd.) determined by suppression subtractive hybridization. Euphytica 196:285-297.

Huang, C.Q., B. Yu, Y.W. Teng, J. Su, Q. Shu, Z.Q. Cheng, and L.Q. Zeng. 2009. Effects of fruit bagging on coloring and related physiology, and qualities of red Chinese sand pears during fruit maturation. Scientia Hort. 121:149-158.

Hu, D., X.B. Liu, H.Z. She, Z. Gao, R.W. Ruan, D.Q. Wu, and Z.L. Yi. 2017. The lignin synthesis related genes and lodging resistance of Fagopyrum esculentum. Biol. Plant. 61:138146. 
Jia, S.R., J.D. Cui, Y. Li, and A.Y. Sun. 2008 Production of L-phenylalanine from transcinnamic acids by high-level expression of phenylalanine ammonia lyase gene from Rhodosporidium toruloides in Escherichia coli. Biochem. Eng. J. 42:193-197.

Jones, K.M., T.B. Koen, S.A. Bound, and M.J. Oakford. 1991. Some reservations in thinning 'Fuji' apples with naphthalene acetic acid (NAA) and ethephon. N. Z. J. Expt. Agr. 19:225-258.

Ju, Z.G. 1998. Fruit bagging, a useful method for studying anthocyanin synthesis and gene expression in apples. Scientia Hort. 77:155-164.

Jun, S.U., Q.X. Meng, L.I. Lin, X. Chen, M.T. Liang, and Q. Shu. 2013. Effect of different light sources on fruit quality of 'Meirensu' Red Chinese sand pear. Northern Hort. 37:5-8.

Kalisz, S., J. Oszmiański, and A. Wojdyło. 2015. Increased content of phenolic compounds in pear leaves after infection by the pear rust pathogen. Physiol. Mol. Plant Pathol. 91:113119.

Keller, M. and G. Hrazdina. 1998. Interaction of nitrogen availability during bloom and light intensity during veraison. II. Effects on anthocyanin and phenolic development during grape ripening. Amer. J. Enol. Viticult. 49:341-349.

Khanal, B.P., E. Grimm, and M. Knoche. 2012. Russeting in apple and pear: A plastic periderm replaces a stiff cuticle. AoB Plants 5:Pls048.

Knobloch, K.H. and K. Hahlbrock. 1977. 4Coumarate:CoA ligase from cell suspension cultures of Petroselinum hortense Hoffm: Partial purification, substrate specificity, and further properties. Arch. Biochem. Biophys. 184:237-248.

Koyama, K., H. Ikeda, P.R. Poudel, and N. GotoYamamoto. 2012. Light quality affects flavonoid biosynthesis in young berries of Cabernet Sauvignon grape. Phytochemistry 78:54-64.

Lafuente, M.T., L. Zacarias, A. Miguel, M.A. Marttı̀nez-Téllez, M.T. Sanchez-Ballesta, and A. Granell. 2003. Phenylalanine ammonialyase and ethylene in relation to chilling injury as affected by fruit age in citrus. Postharvest Biol. Technol. 29:309-318.

Lamb, C.J. and P.H. Rubery. 1975. A spectrophotometric assay for trans-cinnamic acid 4-hydroxylase activity. Anal. Biochem. 68:554 561.

Lefsrud, M.G., D.A. Kopsell, and C.E. Sams. 2008. Irradiance from distinct wavelength lightemitting diodes affect secondary metabolites in kale. HortScience 43:2243-2244.

Legay, S., G. Guerriero, A. Deleruelle, M. Lateur, D. Evers, C.M. André, and J.F. Hausman. 2015. Apple russeting as seen through the RNA-seq lens: Strong alterations in the peel cell wall. Plant Mol. Biol. 88:21-40.

Leyva, A., J.A. Jarillo, J. Salinas, and J.M. MartinezZapater. 1995. Low temperature induces the accumulation of phenylalanine ammonia-lyase and chalcone synthase mRNAS of Arabidopsis thaliana in a light-dependent manner. Plant Physiol. 108:39-46.

Liu, W.H. and S.Y. Cheng. 2003. Effects of the light and mechanical hurt on PAL activities in Ginkgo biloba leaf. Hubei Agricultural Sciences 3:73-75.

Lu, G., Z.J. Li, X.F. Zhang, R. Wang, and S.L. Yang. 2015. Expression analysis of ligninassociated genes in hard end pear (Pyrus pyrifolia Whangkeumbae) and its response to calcium chloride treatment conditions. J. Plant Growth Regul. 34:251-262.

Moura, J.C.M.S., C.A.V. Bonine, J.D.O.F. Viana, M.C. Dornelas, and P. Mazzafera. 2010. Abiotic and biotic stresses and changes in the lignin content and composition in plants. J. Integr. Plant Biol. 52:360-376.

Mertz-Henning, L.M., A.I. Nagashima, F.C. Krzyzanowski, E. Binneck, and F.A. Henning. 2015. Relative quantification of gene expression levels associated with lignin biosynthesis in soybean seed coat. Seed Sci. Technol. 43:445455.

Meyer, C.J., C.A. Peterson, and M.A. Bernards. 2011a. Spatial and temporal deposition of suberin during maturation of the onion root exodermis. Botany-botanique 89:119-131.

Meyer, C.J., C.A. Peterson, and M.A. Bernards. 2011b. A comparison of suberin monomers from the multiseriate exodermis of Iris germanica during maturation under differing growth conditions. Planta 233:773-786.

Morrison, I.M. 1972. A semi-micro method for the determination of lignin and its use in predicting the digestibility of forage crops. J. Sci. Food Agr. 23:455-463.

Rogers, L.A., C. Dubos, I.F. Cullis, C. Surman, M. Poole, J. Willment, S.D. Mansfield, and M.M Campbell. 2005. Light, the circadian clock, and sugar perception in the control of lignin biosynthesis. J. Expt. Bot. 56:1651-1663.

Sanchezballesta, M.T., M.T. Lafuente, L. Zacarias, and A. Granell. 2000. Involvement of phenylalanine ammonia-lyase in the response of Fortune mandarin fruits to cold temperature. Physiol. Plant. 108:382-389.

Santos, J.P.D. and A.F. Wamser. 2006. Fruit bagging effect on the damage caused by biotics and abiotics factors in an organic apple orchard. Rev. Bras. Frutic. 28:168-171.

Shi, C.H., B.X. Qi, X.Q. Wang, L.Y. Shen, L. Jun, and Y.X. Zhang. 2019. Proteomic analysis of the key mechanism of peel russet pigmentation of semi-russet pear under rainwater condition. Scientia Hort. 254:178-186.

Sibout, R., A. Eudes, G. Mouille, B. Pollet, C. Lapierre, L. Jouanin, and A. Séguin. 2005. Cinnamyl alcohol dehydrogenase-C and-D are the primary genes involved in lignin biosynthesis in the floral stem of Arabidopsis. Plant Cell 17:2059-2076.

Sharma, R.R., S.V.R. Reddy, and M.J. Jhalegar. 2014a. Pre-harvest fruit bagging: A useful approach for plant protection and improved post-harvest fruit quality-a review. J. Pomol. Hort. Sci. 89:101-113.

Sharma, R.R., V.R. Sagar, and K. Kumar. 2014b. Effect of pre-harvest fruit bagging on colour, quality and bitter pit disorder in 'Royal Delicious' apple. Bharatiya Vaigyanik evam Audyogi Anusandhan Patrika (BVAAP) 22:53-57.

Teklemariam, T.A. and T.J. Blake. 2004. Phenylalanine ammonia-lyase-induced freezing tolerance in jack pine (Pinus banksiana) seedlings treated with low, ambient levels of ultravioletB radiation. Physiol. Plant. 122(2):244-253.

Tomás-Barberán, F.A. and J.C. Espín. 2001. Phenolic compounds and related enzymes as determinants of quality in fruits and vegetables. J. Sci. Food Agr. 81:853-876.

Villarreal, N.M., C.A. Bustamante, P.M. Civello, and G.A. Martínez. 2010. Effect of ethylene and 1-MCP treatments on strawberry fruit ripening. J. Sci. Food Agr. 90:683-689.

Wang, Y.Z., S.J. Zhang, M.S. Dai, and Z.B. Shi. 2014. Pigmentation in sand pear (Pyrus pyrifolia) fruit: Biochemical characterization, gene discovery and expression analysis with peel pigmentation mutant. Plant Mol. Biol. 85:123-134.

Wang, Y.Z., M.S. Dai, D.Y. Cai, S.J. Zhan, and Z.B. Shi. 2016. A review for the molecular research of russet/semi-russet of sand pear peel and their genetic characters. Scientia Hort. 210:138-142.

Wang, H.Q., O. Arakawa, and Y. Motomura. 2000. Influence of maturity and bagging on the relationship between anthocyanin accumulation and phenylalanine ammonia-lyase (PAL) activity in 'Jonathan' apples. Postharvest Biol. Technol. 19:123-128.

Whiting, P. and D.A.I. Goring. 1982. Chemical characterization of tissue fractions from the middle lamella and secondary wall of black spruce tracheids. Wood Sci. Technol. 16:261267.

Willems, E., L. Leyns, and J. Vandesompele. 2008. Standardization of real-time PCR gene expression data from independent biological replicates. Anal. Biochem. 379:127-129.

Xu, B., L.L. Escamilla-Treviño, N. Sathitsuksanoh, Z.X. Shen, H. Shen, Y.H. Zhang, R.A. Dixon, and B.Y. Zhao. 2011. Silencing of 4-coumarate: Coenzyme A ligase in switchgrass leads to reduced lignin content and improved fermentable sugar yields for biofuel production. New Phytologist 192:611-625.

Zhang, D., B. Yu, J. Bai, M.J. Qian, Q. Shu, J. Su, and Y.W. Teng. 2012. Effects of high temperatures on UV-B/visible irradiation induced postharvest anthocyanin accumulation in 'Yunhongli No. 1' (Pyrus pyrifolia Nakai) pears. Scientia Hort. 134:53-59.

Zhang, Q.F., M.Y. Liu, and J.Y. Ruan. 2017. Metabolomics analysis reveals the metabolic and functional roles of flavonoids in lightsensitive tea leaves. BMC Plant Biol. 17:64. 


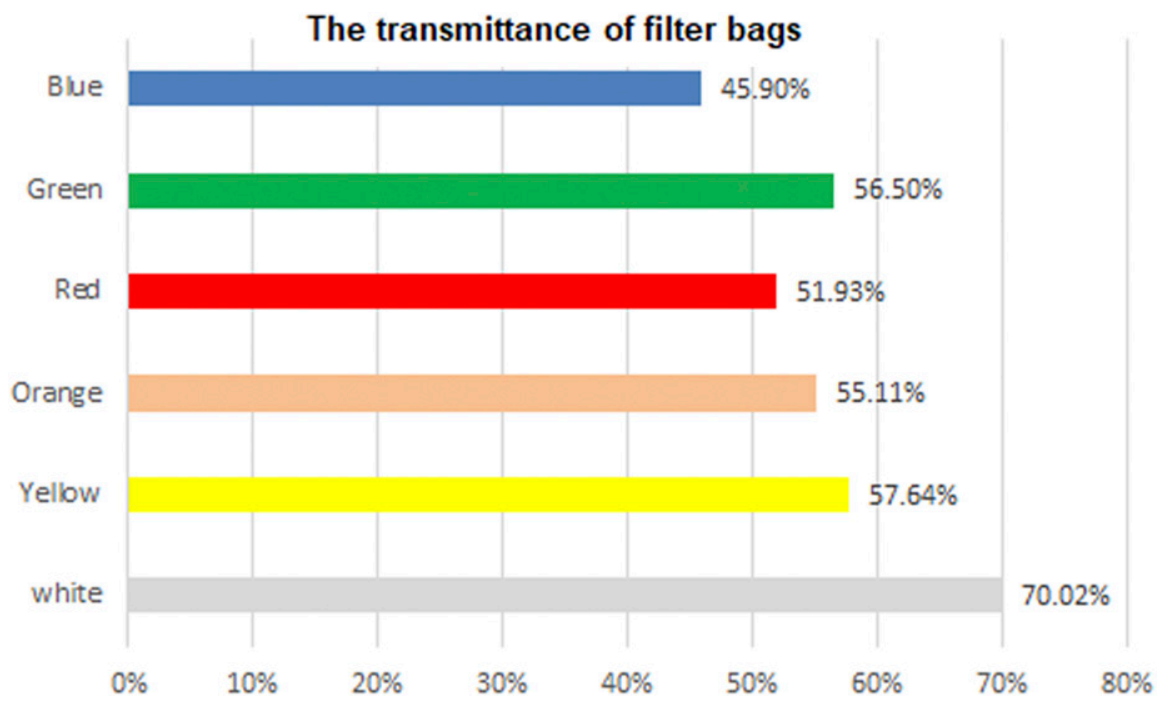

Supplemental Fig. 1. The transmittance of different color of filter bags.

Supplemental Table 1. Primers used for Q-PCR analyses.

\begin{tabular}{|c|c|c|c|}
\hline Gene name & GenBank numbers & Primer name & Primer sequence $\left(5^{\prime}-3^{\prime}\right)$ \\
\hline \multirow[t]{2}{*}{$\overline{P p y P A L 1}$} & FJ478149.1 & PрyPAL1-F & ATTCGTTACTCCACCAAATCCA \\
\hline & & PpyPAL1-R & TTCTCСТCCAAATGCCTCAAA \\
\hline \multirow[t]{2}{*}{ PpyCCR } & GU138672 & $P p C C R-F$ & CAGTCCCTACAAAACAAGAACAAG \\
\hline & & $P p C C R-R$ & CGTGCCCTTTCCTACAAGC \\
\hline \multirow[t]{2}{*}{$\mathrm{PpyC} 4 \mathrm{H}$} & KJ577541.1 & PpyC4H-F & GCCCTGCCAATCCTGGGCATTA \\
\hline & & PpyC4H-R & CATCTTTCTCCCCAAGCACCAATCT \\
\hline \multirow[t]{2}{*}{ PpyCAD } & KX272654.1 & PpyCAD-F & AGGGAGCTCGAACATTTCCAAATGC \\
\hline & & PpyCAD-R & AGGCTTGTCGTCTGCACACTTC \\
\hline \multirow[t]{2}{*}{ Ppy4CL } & KM455955.1 & Ppy $4 C L-F$ & ACGCTGCTGTTGTACCTATGAAGG \\
\hline & & Ppy $4 C L-R$ & AGCCAGCTTTGCTCTCAAGTC \\
\hline \multirow[t]{2}{*}{ PpyPOD } & JQ325052.1 & PpyPOD-F & TCTAGGAGCTCACAGTGTTGG \\
\hline & & PpyPOD-R & TCAGGGCACTTCTTGAGCATG \\
\hline \multirow[t]{2}{*}{ ACTIN } & GU830958 & $A C T I N-F$ & TGGTGTCATGGTTGGTATGG \\
\hline & & $A C T I N-R$ & CAGGAGCAACACGAAGTTCA \\
\hline
\end{tabular}

